\title{
TEE as a monitor during pericardiectomy for constrictive pericarditis
}

\author{
Ramesh Varadharajan • Satyen Parida • Ashok Shankar Badhe
}

Received: 4 January 2014 / Accepted: 5 December 2014 /Published online: 28 December 2014

(C) Indian Association of Cardiovascular-Thoracic Surgeons 2014

Constrictive pericarditis (CP) is a chronic condition where pericardium gets thickened, fibrosed, and calcified, thereby restricting the filling of the ventricles. Surgical removal of the pericardium is the best effective treatment for $\mathrm{CP}$. Because of the advent of newer and better antibiotics, the need for surgical pericardiectomy has declined at least in the developing countries [1]. Nevertheless, malignancy and radiation induced CP is not that uncommon. Pericardiectomy can be either radical or subtotal depending upon the extent of release of adhered pericardium. It can be done via midline sternotomy or anterolateral thoracotomy either on bypass or off bypass. Each technique has got its own merits and demerits.

Two main factors determining the clinical and hemodynamic improvement after surgery are the extent of adequate resection and status of the underlying ventricles. Adequate resection is said to be achieved when both ventricles, atrioventricular grooves, and the bases of the great vessels are freed of the disease process [2]. The adequacy of resection intraoperatively is generally monitored by the fall in the baseline central venous pressures and rarely by the improvement in the voltages of electrocardiogram (ECG) complexes. However, these monitors are not without drawbacks. The central venous pressure monitored by the internal jugular vein (IJV) catheter is influenced by many factors like patient position, volume status, inotrope therapy, anesthetic drugs, and usage of chest retractors. ECG monitor also has got inherent drawbacks like being less sensitive, prone for cautery disturbances, and poor signals owing to loss of contact of electrodes because of the disinfectants used for cleaning, whereas

R. Varadharajan $(\varangle) \cdot$ S. Parida $\cdot$ A. S. Badhe

Department of Anaesthesiology and Critical Care, JIPMER,

Pondicherry, India

e-mail: jipmerramesh@gmail.com

S. Parida

e-mail: jipmersatyen@gmail.com

A. S. Badhe

e-mail: ashokbadhe@hotmail.com trans esophageal echo (TEE) as a monitor has no drawbacks as mentioned above. Intraoperative TEE helps the surgeon in assessing the adequacy of resection and also monitors the dynamic change or improvement in the filling of the ventricles [3].

TEE also helps to guide the surgeon to dissect those left over thickened pericardium even in the least accessible areas. It helps to monitor the dynamic filling of both the ventricles especially the left because the left ventricle being posteriorly located it is difficult to examine the adequacy of expansion by direct visual assessment. Moreover, Doppler assessment of transmitral flow (TMF) and more normalized respiratory variation in TMF should suggest adequate resection [4]. It also serves to sort out the etiology of hemodynamic compromise like damage to the epicardial coronaries reflected by wall motion abnormalities (WMA) should it occur.

To conclude, TEE should be routinely used as a monitor during pericardiectomy. Adequacy of resection should be assessed by TEE especially in right ventricle (RV) inflowoutflow and transgastric mid papillary views apart from hemodynamic assessment.

\section{References}

1. Srivastava AK, Ganjoo AK, Misra B, Chaterjee T, Kapoor A, Pandey CM. Subtotal pericardiectomy via sternotomy for constrictive pericarditis. Asian Cardiovasc Thorac Ann. 2000;8:134-6.

2. Fitzpatrick DP, Wyso EM, Bosher LH, Richardson DW. Restoration of normal intracardiac pressures after extensive pericardiectomy for constrictive pericarditis. Circulation. 1962;25:484-92.

3. Kasravi B, Ng D, Chandraratna PA. Continuous intraoperative transesophageal echocardiography during pericardiectomy for constrictive pericarditis revealing dynamic change in chamber size. Echocardiography. 2005;22:431-3.

4. Skubas N, Beardslee M, Barzilai B, et al. Constrictive pericarditis: intraoperative hemodynamic and echocardiographic evaluation of cardiac filling dynamics. Anesth Analg. 2001;92:1424-6. 